\title{
A comparison of Goldmann and Humphrey automated perimetry in patients with glaucoma
}

\author{
G E TROPE AND R BRITTON \\ From the Department of Ophthalmology, University of Toronto, Toronto General Hospital, Toronto, Ontario, \\ Canada
}

SUMmARY Humphrey automated threshold perimetry (Program 30-2) was performed on 42 eyes of 25 patients with glaucoma to determine both the sensitivity and specificity of automated perimetry in detecting glaucomatous visual field defects. Automated perimetry sensitivity was $90 \cdot 38 \%$, while automated perimetry specificity was $91 \%$. Fifty-two patients and a technician took part in a survey to determine their preference for either test. Patients generally preferred having Goldmann perimetry. The technician favoured Humphrey automated perimetry. Program 30-2 on the automated perimeter took $25 \%$ longer to perform than Goldmann perimetry.

There are a number of automated perimeters on the market. These computerised perimeters all perform visual field tests using preprogrammed and/or programmable test logics. Some of these perimeters can determine retinal threshold sensitivity, and their performance in patients with glaucoma has been favourably compared to that of standard, manual perimetry (Fig. 1).'

The Humphrey automated perimeter is a new and relatively inexpensive automated perimeter that can perform both relatively rapid suprathreshold static perimetry for glaucoma screening and threshold static perimetry. Threshold perimetry allows for quantification and therefore follow-up of patients with glaucomatous visual field defects. Recent reports have indicated that the Humphrey perimeter can be favourably compared with conventional manual perimeters. ${ }^{23}$ We are not, however, aware of studies comparing the sensitivity and specificity of this automated perimeter with standard Goldmann perimetry in patients with glaucoma.

The purpose of this study was two-fold. First we wished to determine patient and technician preference for either machine. Secondly, we wished to determine the sensitivity and specificity of Humphrey automated perimetry using the Goldmann perimeter as standard in patients with glaucoma.

Correspondence to G E Trope, FRCS ed, Toronto General Hospital, Department of Ophthalmology, Glaucoma Division, 200 Elizabeth Street, EN 5-308, Toronto, Ontario, Canada M5G 2 C4.

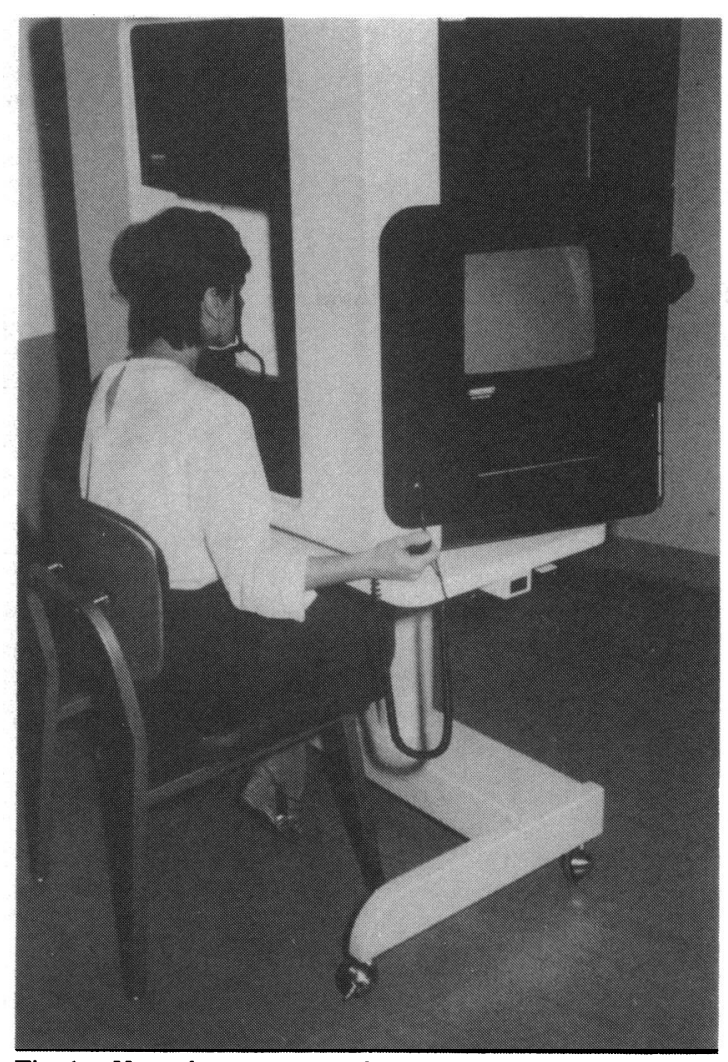

Fig. 1 Humphrey automated perimetry. 


\section{Methods and materials}

This study was divided into two parts.

Part 1. This consisted of a patient and technician survey. Fifty-two glaucomatous patients referred to our visual field service from a number of local ophthalmologists were randomly allocated to Goldmann perimetry and Humphrey automated perimetry on the same day. Program 30-2 was used on the automated perimeter; Goldmann perimetry was performed by standard methods. ${ }^{+}$Both eyes were tested on both machines.

A number of questions were then asked of each patient: (1) Which test did you prefer? (2) With which machine was it easier to keep your eyes straight? (3) Have you ever had a visual field test?

The technician was then asked to fill out a questionnaire answering the following questions: (1) With which perimeter was fixation superior? (2) Which machine was easier to use? (3) Which perimeter would you prefer to use in future on this particular patient?

The mean test time for both eyes on each perimeter was recorded by the technician.

Part 2. One or both eyes of 25 glaucomatous patients attending the Toronto General Hospital Glaucoma Service were tested on both perimeters. Forty-two eyes in total were tested. Patients were randomly allocated to either test. Some of these patients took part in part 1 of the study, but were included in part 2 of the study (visual field comparison study) only if the diagnosis of glaucoma had been absolutely established in at least one eye in all cases by one of us (GT).

\section{INSTRUMENTATION}

Goldmann perimetry was performed by standard techniques. ${ }^{4}$ Humphrey automated perimetry was
Fig. 2 A normal Humphrey visual field.

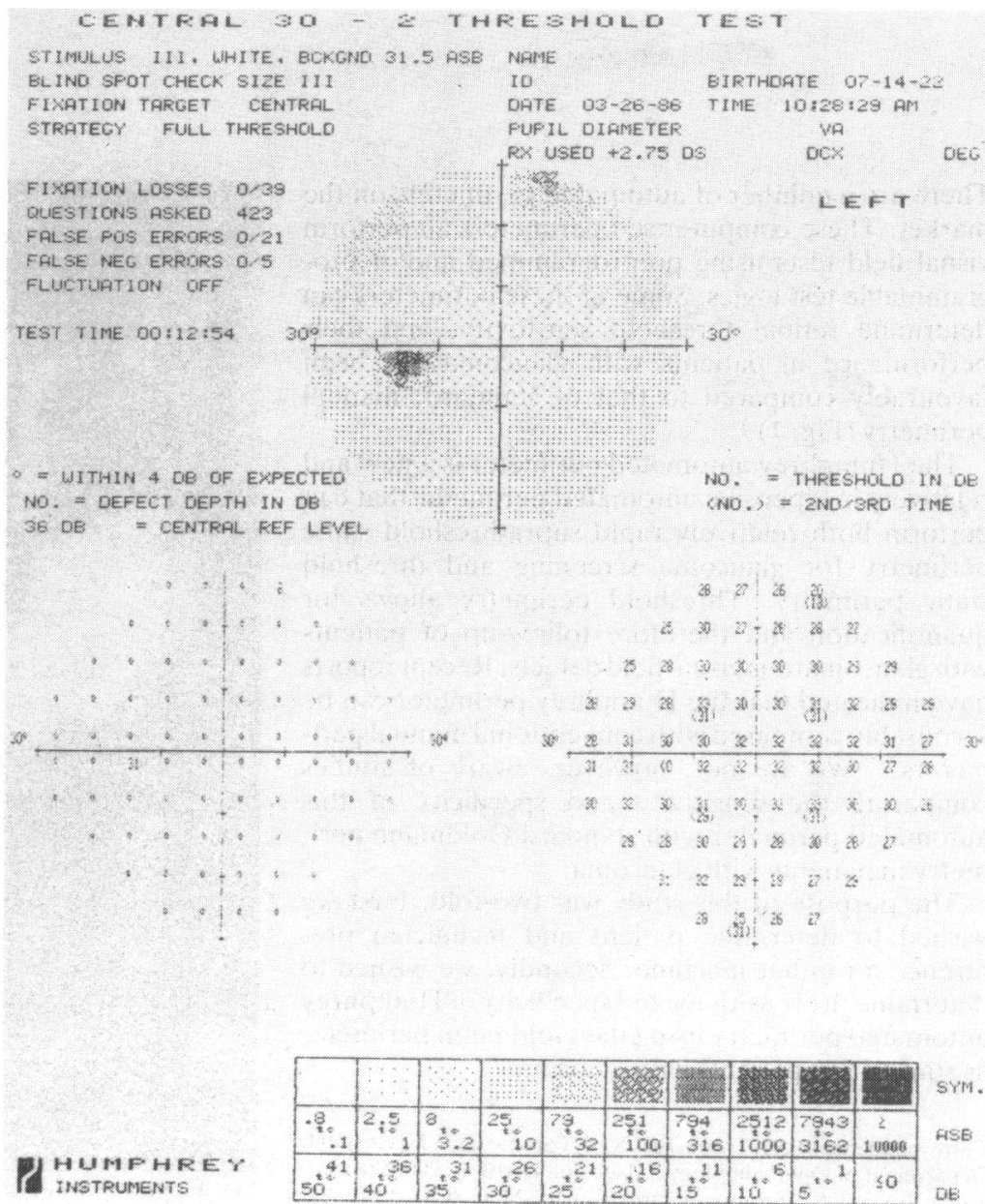


performed with Program 30-2. This program tests the central $30^{\circ}$ field at 76 points. Points tested are $6^{\circ}$ apart. Initially, this automated perimeter determines retinal threshold at four primary points-i.e., one in each quadrant $10^{\circ}$ from fixation. It does so by presenting a stimulus which is very bright (suprathreshold). The intensity is then reduced in $4 \mathrm{db}$ decrements until it is not seen. The stimulus then increases in $2 \mathrm{db}$ increments until it is seen again. This value is recorded as the patient's retinal threshold. The four initial thresholds are then used as starting points to determine the thresholds of neighbouring points by means of the same bracketing technique. These points are used in a similar manner to determine retinal thresholds of neighbouring points until the central $30^{\circ}$ area has been fully tested. If a value varies from expected by more than $4 \mathrm{db}$, it is remeasured automatically.

The Humphrey automated perimeter Program 30-2 uses a background illumination of 31.5 apostilbs with a $4 \mathrm{~mm}^{2}$ stimulus size (equivalent to Goldmann perimeter size III target); the appropriate near correction for age is used.

The nasal step was not tested in this study as the nasal step program had not yet been installed in the perimeter. Fixation is monitored both by the technician and indirectly by the machine.

The blind spot is located at the beginning of the test, and during the test approximately $10 \%$ of the stimuli are presented in the blind spot. If fixation is accurate, the patient will not see any of the stimuli presented in the region of the blind spot. If the patient responds that he does see a significant number of stimuli presented to the blind spot, a beeping sound is emitted by the machine to alert the technician to encourage patient fixation. The number of times fixation is lost is typed out on the result sheet. The test continues to run even if fixation is very poor.

The machine also assesses patient reliability by occasionally producing just the sound associated with the light stimulus without actually presenting the light. If the patient responds to this sound, by pressing the response button, this is considered a false positive result. Periodically a suprathreshold stimulus is presented to the patient that he/she should easily see. If the patient fails to respond, this indicates lack of attention, a false negative result. Fixation loss and false positives and negatives are all printed out on the visual field chart together with a grey scale, decibel scale, and a scale that indicates deviation of retinal threshold from expected decibel values (Fig. 2).

ANALYSIS OF VISUAL FIELDS

For part 2 of this study (sensitivity and specificity) we used decibel criteria modified from papers published by Heijl and Drance. ${ }^{15}$ The criteria for abnormality with the Humphrey automated perimeter were: (1) All peripheral test points were excluded to avoid lens rim artefact. (2) Test points immediately above and below the blind spot were excluded. (3) A $5 \mathrm{db}$ difference between two neighbouring points in conjunction with a $10 \mathrm{db}$ difference in mirror image points between the upper and lower hemifield were considered positive glaucoma visual field defects. (4) One or more points $15 \mathrm{db}$ below the point of highest retinal sensitivity was considered an abnormal visual field.

Sensitivity and specificity results were analysed according to standard methods. ${ }^{6}$ The sensitivity of Humphrey automated perimetry was defined as the number of abnormal Humphrey visual fields divided by the number of Goldmann abnormal visual fields expressed as a percentage. Humphrey automated perimetry specificity was determined by dividing the number of normal Humphrey automated perimetry fields by the number of normal Goldmann visual fields expressed as a percentage.

\section{Results}

Part 1. Table 1 reviews the results of the patients surveyed. Sixty per cent of the patients preferred Goldmann perimetry. Patients generally thought fixation was easier to maintain with the Goldmann perimeter. Fifty four per cent of patients, however, had previously had a Goldmann visual field test. None of the patients had previously undergone automated visual field testing.

Table 2 reviews the results of the technician survey. Fixation appeared to be similar to the technician during both of the tests. The technician found automated perimetry overall a little easier to perform than Goldmann perimetry. Technician preference strongly favoured Humphrey perimetry.

The mean test time for both eyes was $26 \cdot 2$ minutes

Table 1 Fifty-two patient responses

\begin{tabular}{llll}
\hline & Goldmann & Humphrey & Same \\
\hline Patient preference & $60 \%$ & $17 \%$ & $23 \%$ \\
Fixation ease & $44 \%$ & $21 \%$ & $35 \%$ \\
Previous field test & $54 \%$ & $0 \%$ & - \\
\hline
\end{tabular}

Table 2 Technician survey

\begin{tabular}{llll}
\hline & Goldmann & Humphrey & Same \\
\hline Fixation & $15 \%$ & $15 \%$ & $70 \%$ \\
Technical ease & $10 \%$ & $40 \%$ & $50 \%$ \\
Preference & $13 \%$ & $67 \%$ & $20 \%$ \\
\hline
\end{tabular}


Table 3 Sensitivity and specificity of Humphrey perimetry using Goldmann perimetry as standard

Humphrey automated perimetry sensitivity:

$$
\frac{28}{31} \times \frac{100}{1}=90.3 \%
$$

Humphrey automated perimetry specificity:

$$
\frac{10}{11} \times \frac{100}{1}=91 \%
$$

for Goldmann perimetry and 32.6 minutes for automated perimetry.

Part 2. Table 3 reviews the sensitivity and specificity of Humphrey automated perimetry Program 32.

Sensitivity tests showed that the Humphrey automated perimeter detected 28 abnormal glaucomatous visual fields out of a total of 31 abnormal fields $(90 \cdot 3 \%)$. Specificity tests showed that there were 10 normal Humphrey fields compared with 11 normal Goldmann fields (91\%).

\section{Discussion}

The major purpose of this study was to determine the sensitivity and specificity of Humphrey automated perimetry to detect glaucomatous visual field defects using the Goldmann perimeter as the standard. The results of this section of the study indicate that Program 30-2 is both highly sensitive and specific for detecting glaucomatous visual field defects. The sensitivity and specificity results reported here are similar to those reported for the Octopus perimeter using Tübinger perimetry as the standard.'

Although the Humphrey automated perimeter is both sensitive and specific for glaucomatous visual field defects, it does have various drawbacks when compared with Goldmann perimetry. Firstly, auto-
Fig. 3 An abnormal Humphrey visual field.

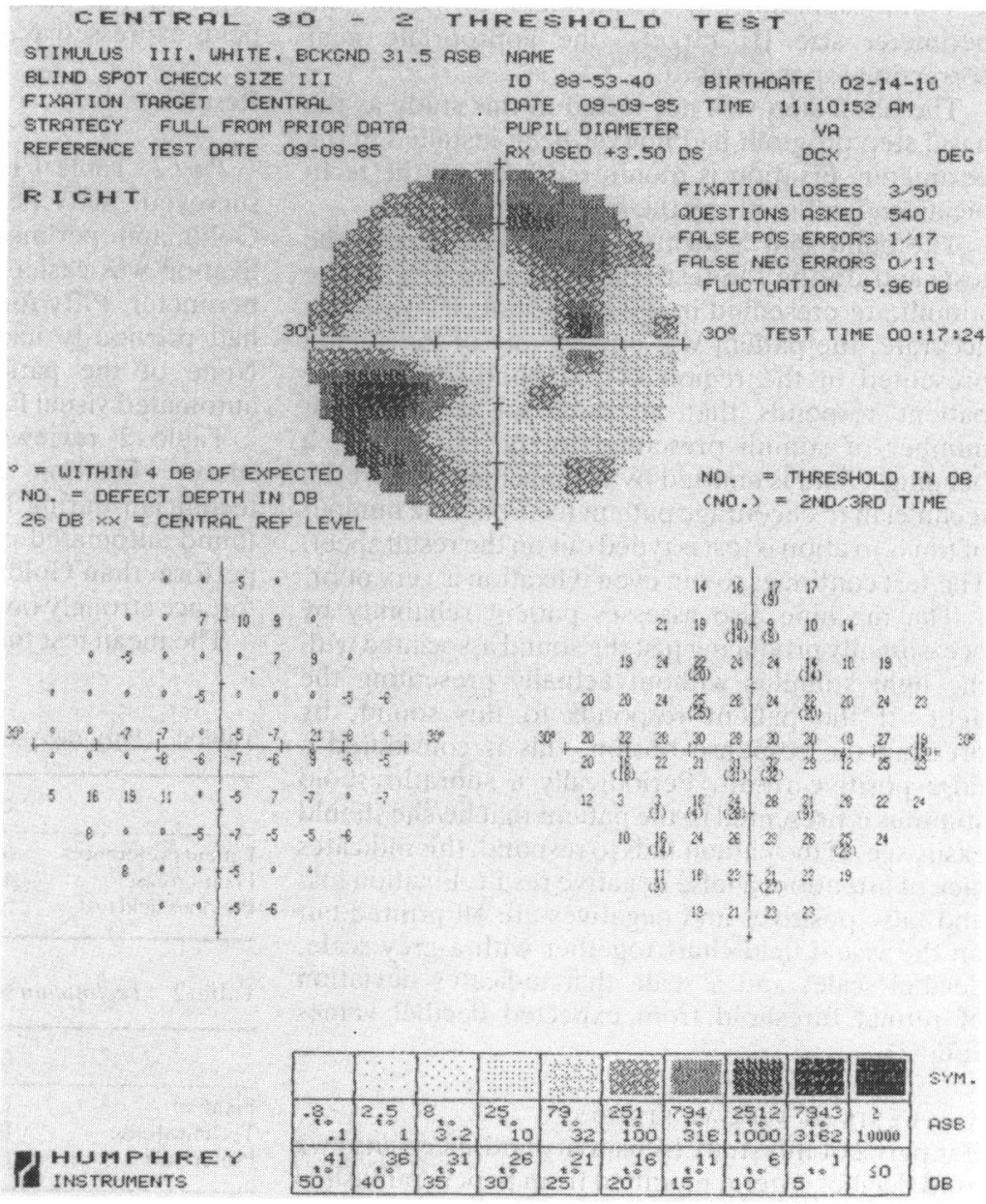


mated perimetry takes about $25 \%$ longer to perform than Goldmann manual perimetry. Further, patients clearly prefer Goldmann perimetry to automated perimetry, but this preference may be biased by the fact that nearly half the patients had previously undergone only Goldmann perimetry. The prolonged test time, the rapid appearance and disappearance of the light stimulus, and the fact that the test continues to run despite detection of poor fixation suggest to us that this automated perimeter in no way overcomes many of the major obstacles to accurate visual field assessment such as accurate fixation, maintenance of concentration, and patient fatigue. A major advantage of the Humphrey automated perimeter, however, is that it can be performed by less highly trained technicians. It also overcomes the tedium of manual perimetry, and operator error is completely avoided. ${ }^{s}$

It is important to note that the use of only Program 30-2 on the Humphrey automated perimeter, although highly sensitive and specific for glaucomatous visual field defects, will not detect visual field defects close to fixation nor in the nasal step area beyond $30^{\circ}$. Furthermore, it will not detect defects between the 76 points tested in the central $30^{\circ}$ (Fig. 3 ). The machine does, however, have a separate program to test the nasal step area and a macular threshold program to test the visual field close to fixation. Static profiles can also be tested. Other programs also exist which allow one to test areas not tested by Program 30-2. Clearly, however, the use of Program 30-2 with one or more of these other test programs will add significantly to the already fairly lengthy test period. These combined programs will, however, allow for very accurate assessment of the visual field in glaucoma.

We thank Mrs Mena Cali and other technicians at the Toronto General Hospital for performing visual field tests. We also thank Dr Clive Mortimer for his help and Mrs Gail Taylor-Cole for typing the manuscript.

\section{References}

1 Heijl A, Drance SM. A clinical comparison of three computerized automatic perimeters in the detection of glaucoma defects. Arch Ophthalmol 1981; 99: 832-6.

2 Beck RW, Bergstrom TJ, Lichter PR. A clinical comparison of visual field testing with a new automated perimeter, the Humphrey field analyzer and the Goldmann perimeter. Ophthalmology 1985; 92: 77-82.

3 Mogil LG, Abramovsky-Kaplan I, Rosenthal S, Podos SM. Comparison of Goldmann, Humphrey and Octopus perimetry in glaucoma. Invest Ophthalmol Vis Sci 1985; 26: 225.

4 Rock WJ, Drance SM, Morgan RW. A modification of Armaly visual field screening technique for glaucoma. Can J Ophthalmol $1981 ; 6: 283-90$.

5 Heijl A. Computerized perimetry. Trans Ophthalmol Soc UK 1984; 104: 76-87.

6 Sommer A. Epidemiology and statistics for the ophthalmologist. New York: Oxford University Press, 1980: 11.

Accepted for publication 27 August 1986. 